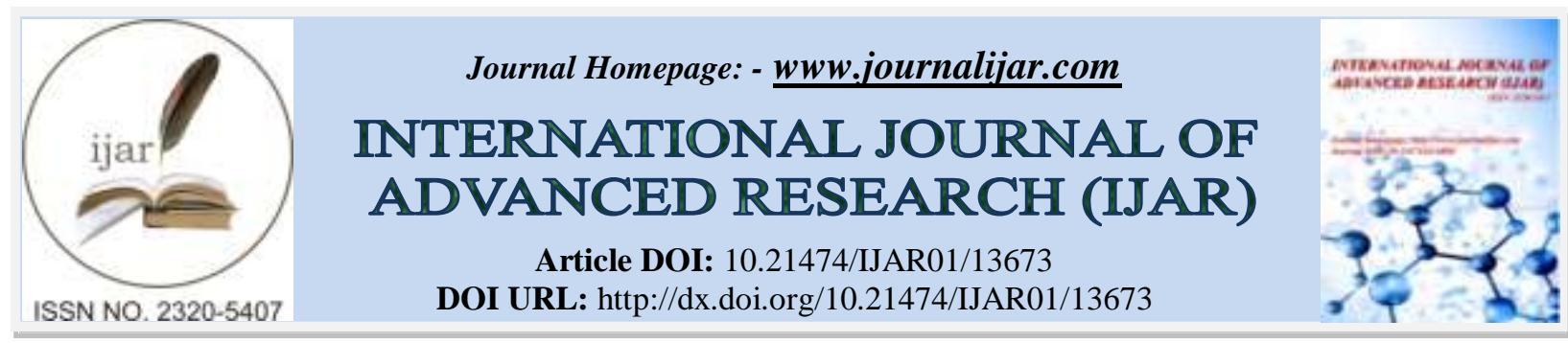

RESEARCH ARTICLE

\title{
LE PROFIL EPIDEMIOLOGIQUE ET LA PRISE EN CHARGE DES PERFORATIONS GRELIQUES POST-TRAUMATIQUES A PROPOS DE 50 CAS
}

Saad Mahamoud, Khalid Rabbani and Abdeloua Hed Louzi

CHU Mohamed VI, Université Caddi Ayyad, Marrakech, Maroc.

\section{Manuscript Info}

Manuscript History

Received: 31 August 2021

Final Accepted: 30 September 2021

Published: October 2021

\begin{abstract}
Les perforations grêliques post traumatique plus fréquents chez les jeunes sont de complications tardives et rare dans les traumatismes de l'abdomen fermées. La disponibilité d'un plateau technique performant constitue classiquement une des principales conditions pour la réussite de cette attitude. Nous essayons d'étudier les différents aspects épidémiologiques, diagnostiques et thérapeutiques de cette affection à travers les résultats d'une série de 50 malades. Une série de 50 patients est prise en charge par le traitement opératoire. La gravité des lésions est appréciée par les données cliniques et paracliniques, ainsi que la morbidité et la mortalité sont analysées. Les accidents de la circulation étaient la première cause des perforations grêliques, suivi par les plaies par arme blanche (34\% cas) dans notre contexte. Les épanchements intrapéritonéaux étaient les plus fréquentes (30\% des cas). La notion de polytraumatisme était très fréquente. La durée moyenne de surveillance était de 7 jours. Une laparotomie en urgence était indiquée chez tous les patients. On avait noté un taux de mortalité de $3.7 \%$ soit 4 cas. Il semble à partir de notre expérience que la rapidité de la prise en charge pluridisciplinaire peut constituer une référence thérapeutique dans les pays occidentaux comme dans les pays en voie de développement, sûre et justifiée.
\end{abstract}

Copy Right, IJAR, 2021,. All rights reserved.

\section{Introduction:-}

Les accidents de la circulation font partie des causes principales des traumatismes de l'abdomen aussi bien dans les pays occidentaux que les pays en développement. [4]

Les atteintes de la grêle lors des traumatismes abdominaux ont trois caractéristiques essentielles qui font toute la difficulté de la prise en charge des patients qui en sont atteints :

- Leur symptomatologie est retardée, peut être masquées par les effets thérapeutiques ou au contraire simulée d'associations lésionnelles.

-leur conséquence ultime est la péritonite généralisée engageant le pronostic vital chez le sujet sain et vient sérieusement obérer le pronostic du polytraumatisé. [5]

Corresponding Author:- Saad Mahamoud

Address:- CHU Mohamed VI, Université Caddi Ayyad, Marrakech, Maroc. 
La radiographie de l'abdomen sans préparation ASP met en évidence la présence d'une grisaille diffuse et/ou d'un pneumopéritoine. [6]

Dans le cas douteux, l'échographie et scanner peuvent porter le diagnostic en mettant en évidence un liquide intra péritonéal. [6] Le pronostic dépend de la gravidité des lésions initiales mais ausside la rapidité de la prise en charge

\section{Matériel Et Méthodes:-}

Ce travail a porté sur 50 cas des perforations grêliques post traumatique, hospitalisés au service de chirurgie générale sur une période de huit ans allant du ler janvier 2008 à la fin décembre 2016.Les dossiers des patients ont été analysés selonune fiche d'exploitation préétablie.

\section{Résultats:-}

La série globale comportait 50 cas. L'âge moyen était de 32 ans (extrêmes 17 à 73 ans). Il y avait 46 hommes (92\%) et 4 femmes (8\%). Les circonstances du traumatisme (Figure 1), étais dominés par les accidents de la voie publique (46\%) suivis les plaies par armes blanche (34\%), des accidents de sport (12 $\%)$, des chutes de hauteurs (8\%) et. Le mécanisme était direct chez les patients dont la majorité sont urbaines le motif d'admission avec une contusion abdominale de $62 \%$,plaies pénétrante $32 \%$ et une éviscération de $2 \%$.

Quatre patients étaient en état dechoc et 3 patients en détresse respiratoire. . Les signes fonctionnels étaient dominés par la douleur qui était abdominale dans 16 cas et basithoracique dans 4 cas. Les autres signes fonctionnels étaient des vomissements chez 14 patients (13\%), une distensionabdominale chez 5 patients $(5 \%)$ et une hémorragie digestive (1 cas). L'examen avait montré une défense abdominale chez $32 \%$ desmalades (16 cas) et une sensibilité abdominale chez 60\% d'entre eux (Tableau 1). Un polytraumatisme était retrouvé chez $48 \%$ des patients (Figure 2), avec des traumatismes des membres (18 cas), thoracique (5 cas) et bassin ( 1 cas). Le bilan biologique avait trouvé une anémie normochrome dans 40 cas, une cytolyse dans 26 cas et une cholestase dans 4 cas. La radiographie d'abdomen sans préparation avait objectivé des niveaux hydroaériques dans 4 cas et un pneumopéritoine dans 2 cas, alors que la radiographie du thorax avait montré un épanchement pleural dans 10 cas, des fractures de côtes dans 6 cas, des foyers de contusion pulmonaire dans 3 cas et un pneumothorax dans 3 cas. L'échographie abdominale était réalisée en urgence chez tous les patients. Elle avait montré un épanchement intrapéritonéal dans 30 cas (de moyenne abondance dans 51 cas) et des lésions viscérales dans 60 cas. Il s'agissait de lésions hépatiques dans 27 cas (16 contusions, 6 fractures et 5 hématomes) et des lésions spléniques dans 21 cas (14 contusions, 5 fractures et 2 hématomes) (Tableau 2). Un scanner abdominal était réalisé dans 30 cas (60\%). Il avait montré un épanchement intra péritonéal dans 15 cas, un épanchement pleural dans 5 caset un pneumopéritoine dans 2 cas. Les lésions hépatiques à type de contusion hépatique dans 4 cas, fracture hépatique dans 4 cas et hématome sous capsulaire dans 2 cas. Les lésions spléniques étaient retrouvées chez 3 patients. La prise en charge de ces patients était primaire pour (75.4\%) et par transfert pour (24.6\%). Une transfusion sanguine de culots globulaires était nécessaire chez 10 de nos patients, et le nombre moyen de culots globulaires (CG) était de 3,5 culots par patient. Une laparotomie en urgence était indiquéechez tous les patients (100\%) devant l'instabilité hémodynamique malgré les mesures de réanimation dans 7 cas, des signes de péritonite avec la présence de pneumopéritoine et de contracture abdominale . On avait noté un taux de mortalité de $3.7 \%$ soit 4 cas. Des complications étaient survenues chez 7 patients avec 2 cas de bilome, 3 cas d'hémobilie a2 cas de surinfection de la plaie chirurgicale. L'évolution était jugée favorable chez 3patients avec une durée moyenne d'hospitalisation de 7 jours.

\section{Discussions:-}

Les perforations grêliques post traumatique sont un défi diagnostique et thérapeutique pour le médecin, le traumatisme fermé présente un taux de mortalité induit plus important du fait qu'il est difficile à diagnostiquer et qu'il est souvent associé à d'autres traumatismes intra- ou extrapéritonéaux [2,3].

Les données épidémiologiques générales de notre population semblent être concordantes avec les données de la littérature. Celle-ci montre que les contusions abdominales touchent en premier le sujet jeune, de sexe masculin [4, 5]. Les AVP constituent la première étiologie, suivis des chutes d'un lieu élevé [6-8]. La douleur constitue un signe d'appel primordial dans les contusions abdominales, elle est souvent localisée suivant la projection del'organe lésé sur la paroi abdominale. 
Les vomissements témoignent d'une irritation péritonéale. La distension abdominale, l'hémorragie digestive ainsi que l'hématurie sont d'autres signes fonctionnels à rechercher de façon systématique. L'examen clinique, malgré ses insuffisances et ses difficultés de réalisation, est jugé fondamental par la plupart des auteurs permettant d'apprécier: la fonction ventilatoire, température, recherche de douleurs, météorisme, trouble du transit, signes d'anémie, mesure du pouls, de la tension, de la diurèse [9]. Sur le plan biologique, les principaux examens à prescrire sont : l'hématocrite, l'hémoglobine, la numération formule sanguine, un bilan hépatique, le dosage des lipases ou des amylases. L'échographie constitue un outil essentiel permettant de faire le diagnostic des collections liquidiennes intra et rétro péritonéales et celui des lésions d'organes pleins ainsi que leur surveillance en cas de traitement conservateur [9]. Le lavage péritonéal peut être une autre option possible en l'absence d'échographie ou si l'état d'un patient est instable, mais il est de moins en moins pratiquer [10]. Quant à la tomodensitométrie abdominale avec contraste intraveineux, elle est devenue l'examen de référence pour le diagnostic et le suivi des patients présentant des traumatismes abdominaux importants. L'angiographie demeure un élément d'évaluation en second plan, parfois essentielle pour confirmer une hémorragie active ou retardée et pour procéder à l'embolisation, au besoin [11].

Au terme de ces examens, l'identification du bilan lésionnel permet de distinguer des lésions élémentaires et des lésions spécifiques. La chirurgie en urgence reste la règle chez le traumatisé abdominal dont l'hémodynamique est instable malgré une réanimation bien menée, ou en cas de lésion évidente d'organe creux. La laparotomiepar voie médiane sera dans ce cas préférable. La mini laparotomie représente 1'alternative entre l'exploration par une laparotomie classique et la laparoscopie. Elle n'est plus de mise si cette dernière peut être réalisée. La laparotomie écourtée correspond à la réalisation d'un geste le plus rapide possible et donc incomplet, limité au constat des lésions et au contrôle sommaire d'une hémorragie active et/ou d'une fuite digestive, suivis de la fermeture de la laparotomie pour laisser la place au plus vite à la réanimation qui s'impose [12]. Parallèlement, certaines études récentes révèlent le succès du traitement conservateur des contusions abdominales chez l'adulte. Il est réalisable dans 55\% à $80 \%$ des cas avec un tauxde réussite de l'ordre de $60 \%$ ou 70\% [13], dans l'optique de diminuer les interventions chirurgicales et ainsi d'augmenter leschances de préserver les organes pleins, avec possibilité d'intervenir secondairement, pour permettre une prise en charge moins effractive d'un traumatisme abdominal. Cette option nécessite une surveillance chirurgicale et médicale clinique vigilante du traumatisé de l'abdomen initialement non opéré, quelque soit l'organe atteint [14]. Cette surveillance « armée » comporte la disponibilité d'une équipe de chirurgiens, d'anesthésistes et de radiologues, disposant d'outils diagnostiques performants. L'approche non opératoire est loin d'être non chirurgicale : à tout moment, le blessé peut présenter des signes évoquant la poursuite d'une hémorragie, une atteinte d'organe creux ou du pancréas, un syndrome ducompartiment abdominal [15,16]. Ses indications augmentent grâce à l'apport de l'embolisation par voie artérielle. Cette technique a été initialement développée pour les patients atteints d'un traumatisme retropéritonéal grave, essentiellement rein et bassin, et elle est maintenant réalisable chez des patients présentant des traumatismes hépatiques et, plus récemment, spléniques. Les échecs du traitement par observation surviennent principalement au cours des premières 24 heures [17,18]. Cette donnée pourrait être à prendre en compte pour l'orientation de l'observation des patients.X

\section{Conclusion:-}

Les perforations grêliques lors d'un traumatisme fermé de l'abdomen sont des urgences chirurgicaux dont la prise en charge doit être rapide en mettant une collaboration multidisciplinaire efficace associant des équipes médicales, chirurgicales et radiologiques afin de conserver le pronostic vital.

\section{Conflits d'intérêts}

Les auteurs déclarent qu'ils n'ont aucun conflit d'intérêts

\section{Tableaux et figures}

Tableau 1: les différents éléments de l'examen abdominal Tableau 2: les différentes lésions viscérales au cours des perforations post traumatismes au scanner

Figure 1: étiologies des perforations grêliques posttraumatisme

Tableau 3: lésions associées à la perforation grêlique 
Tableau I:- Les différents éléments de l'examen abdominal.

\begin{tabular}{|l|c|c|c|}
\hline \multicolumn{2}{|c|}{ Signes physiques } & Nombre & Pourcentage(\%) \\
\hline \hline \multirow{2}{*}{ Distensionabdominale } & 1 & $2 \%$ \\
\hline \multirow{2}{*}{ Sensibilitéabdominale } & Localisé & 20 & $40 \%$ \\
\cline { 2 - 4 } & Généralisé & 10 & $20 \%$ \\
\hline \multirow{2}{*}{ Défenseabdominale } & Localisé & 10 & $20 \%$ \\
\cline { 2 - 4 } & Généralisé & 6 & $12 \%$ \\
\hline \multirow{2}{*}{ Contractureabdominale } & & 2 & $4 \%$ \\
\hline \multirow{2}{*}{ Ecchymoses,hématomes } & 20 & $40 \%$ \\
\hline
\end{tabular}

Tableau 2:- Résultats du scanner thoraco-abdomina( au cours des perforations, grêliques.

\begin{tabular}{|l|c|c|}
\hline \multicolumn{1}{|c|}{ Résultats du scanner } & Nombre de cas & Pourcentage (\%) \\
\hline Epanchement intra-péritonéal & 15 & 30 \\
\hline Epanchementpleural & 5 & 10 \\
\hline Pneumopéritoine & 2 & 4 \\
\hline Contusionhépatique & 4 & 8 \\
\hline Fracturedufoie & 4 & 6 \\
\hline Contusionsplénique & 3 & \\
\hline
\end{tabular}

Figure 1:- Répartition des patients selon les étiologies.

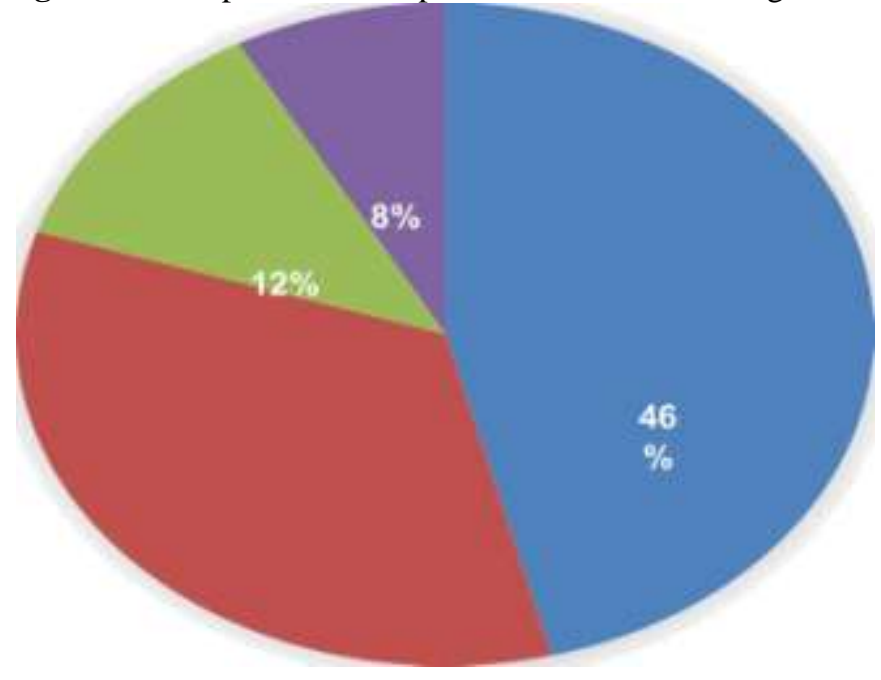


"AVP Plaie par arme blancheAccident de sport

"Chute d'une hauteur

Tableau 3:- Les différents traumatismes associés au cours des perforationsGréliques.

\begin{tabular}{|l|c|c|}
\hline Traumatisme associé & Nombre de cas & Pourcentage \\
\hline Lesmembres & 18 & 36 \\
\hline Thorax & 5 & 10 \\
\hline Bassin & 1 & 2 \\
\hline
\end{tabular}

\section{Références:-}

1. Menegaux F. Lésions abdominales traumatiques. Encycl Méd Chir Urgences, ed ESeME SAS. 2003; 24-105 -A-30. GoogleScholar

2. Arvieux C. Non-operative management of blunt splenic trauma in the adult. J Chir. 2008;145(6):531. PubMed | Google Scholar

3. Engelhardt S, Hoyt D, Coimbra R, Fortlage D, Holbrook T. The 15- year evolution of an urban trauma center: what does the future holdfor the trauma surgeon. J Trauma. 2001 ; 51(4) : 633-7. PubMed Google Scholar

4. Acierno SP, Jurkovich GJ, Nathens AB. Is pediatric trauma stilla surgical disease? Patterns of emergent operative interventionin the injured child. J Trauma. 2004 ; 56 (5) : 960-4. PubMed Google Scholar

5. Letoublon C, Chen Y, Arvieux C, et al. Delayed Celiotomy or Laparoscopy as part of the Nonoperative Management of Blunt Hepatic Trauma. World J Surg. 2008;32(6):1189-93. PubMed Google Scholar

6. Arvieux C, Letoublon C. La laparotomie écourtée pour le traitement des traumatismes abdominaux sévères: principes de technique et de tactique chirurgicales. Annales de chirurgie. 2006 ;131(5): 342-346. PubMed | Google Scholar

7. Miller LA, Shanmuganathan K. Multidetector CT evaluation of abdominal trauma.Radiol Clin North Am. 2005;43(6): 1079-95. PubMed | Google Scholar

8. Bonnet F, Fulgencio JP, Guerrini P. Apport de 1 'imagerie en pathologie traumatique abdominale. 1996. Paris. Elsevier (Conférences d'actualisation SFAR). Google Scholar

9. Thomas V, Ragozzino W, Ramshaw D, Churchill P, Covington D, Maxwell G. Oral contrats is most necessary in the evaluationof blunt abdominal trauma by computed tomography. Am J Surg. 1993 , 166(6): 680-89. PubMed | Google Scholar

10. Baranger B, Vicq P. Contusions et plaies de l'abdomen : Traumatismes de la rate. 1995. Paris. Encycl Med Chir Gastroentérologie. Fa 9-007- A-10. Google Scholar

11. Myers JG, Dent DL, Stewart RM, Gray GA et coll. Blunt splenic injuries: dedicated trauma surgeons can achieve a high rate of nonoperative success in patients of all ages. J Trauma. $2000 ; 48$ (5) ' 801-5. PubMed | Google Scholar

12. Santucci RA, McAninch JW, Safir M, Mario LA et coll. Validation of the American Association for the Surgery of Trauma organ injury severity scale for the kidney. J Trauma. 2001 ; 50 (2) 195-200. PubMed | Google Scholar

13. Haan JM, BifflW, Knudson MM et coll. Splenic embolization revisited: a multicenter review. J Trauma. 2004 ; 56 (3) : 542- PubMed | Google Scholar

14. Sclafani SJ, Shaftan GW, Scalea TM et coll. Nonoperative salvage of computed tomography-diagnosed splenic injuries: utilization of angiography for triage and embolization for hemostasis. J Trauma. 1995 ; 39 (5) : 818 -25. PubMed | Google Scholar

15. Peitzman AB, Heil B, Rivera L et coll. Blunt splenic injury in adults: Multiinstitutional Study of the Eastem Association for the Surgery of Trauma. J Trauma. 2000 ; 49 (2)177-87. PubMed |Google Scholar

16. Haan JM, Bochicchio GV, Kramer N, Scalea TM. Nonoperative management of blunt splenic injury: a 5 -year experience. J Trauma. 2005;58(3):492-8. PubMed | Google Scholar

17. Peitzman AB, Heil B, Rivera L, et al. Blunt splenic injury in adults: Multi-institutional Study of the Eastern Association for the Surgery of Trauma. J Trauina. 2000;49(2):177-87.PubMed | Google Scholar

18. Brugère C, Arvieux C, Dubuisson V, et al. Early embolisation in the non-operative management of blunt splenic injuries: aretrospective multicenter study. J Chir. 2008;145(2): 126-32. PubMed | Google. 